\title{
Efeito do uso de minerais traços (cobre, zinco, selênio e manganês) e vitaminas (A e E) injetáveis sobre o estresse oxidativo e função de neutrófilos de fêmeas da raça Holandesa no período de transição
}

[Effect of use of injected trace minerals (copper, zinc, selenium, and manganese) and vitamins (A and E) on oxidative stress and neutrophil function in females of Hostein cows during transitionl ]

\author{
S.G. Collet $^{1}$, J.A. Ferronatto ${ }^{2}$, M. Santos ${ }^{3}$, M.G. Blagitz ${ }^{3}$, M.S.P. Enders ${ }^{1}$, \\ E.I. Müller ${ }^{1}$, R.S. Sousa ${ }^{4}$, E.L. Ortolani ${ }^{4}$, A. Thaler Neto ${ }^{5}$, M.L.R. Leal ${ }^{1}$ \\ ${ }^{1}$ Universidade Federal de Santa Maria - Santa Maria, RS \\ ${ }^{2}$ Universidade do Oeste de Santa Catarina - Xanxerê, SC \\ ${ }^{3}$ Universidade Federal da Fronteira Sul - Realeza, PR \\ ${ }^{4}$ Universidade de São Paulo - São Paulo, SP \\ ${ }^{5}$ Universidade do Estado de Santa Catarina - Lages, SC
}

Silvana Giacomini Collet

https://orcid.org/0000-0002-1391-9468

José Augusto Ferronatto

https://orcid.org/0000-0001-8409-745X

Michele Santos

https://orcid.org/0000-0003-0188-1527

Maiara Garcia Blagitz

https://orcid.org/0000-0002-4544-7530

Edson Muller

https://orcid.org/0000-0002-0884-1774

\section{RESUMO}

O objetivo deste estudo foi avaliar o efeito metafilático de minerais traços e vitaminas A e E injetáveis, em parâmetros do estresse oxidativo e na função de neutrófilos em fêmeas da raça Holandesa no período de transição. Sessenta animais foram divididos em dois grupos: animais que receberam minerais traços e vitaminas A e E injetáveis (GMTV) $(n=30)$ e grupo controle $(\mathrm{GC})(\mathrm{n}=30)$. Não houve diferença significativa entre os grupos nos parâmetros avaliados, porém observou-se diferença significativa entre tratamento e dia para os valores da SOD, com maior atividade dessa enzima em fêmeas GMTV, nas semanas próximas ao parto. Observou-se diferença de dia e para interação tratamento e dia para o TBARS, em que fêmeas GMTV mostraram menores valores de TBARS em todos os momentos, exceto sete dias após o parto. Houve efeito significativo de dia para leucócitos, interação tratamento e dia para neutrófilos e interação tratamento e dia para fagocitose de neutrófilos, em que as fêmeas GMTV apresentaram valores menores de leucócitos e neutrófilos próximo ao parto, além de maior fagocitose de neutrófilos. Pode-se observar que houve melhora no sistema oxidativo e imune de fêmeas GMTV, resposta que provavelmente está relacionada com a administração dos minerais traços e vitaminas A e E.

Palavras-chave: superóxido dismutase, glutationa peroxidase, TBARS, NBT, período de transição

\begin{abstract}
This study evaluates the metaphylactic of the subcutaneous administration of a trace minerals and vitamins $A$ and $E$, on the oxidative stress and neutrophil function in Holsteins cows under the transition period. Sixty females were divided in two groups: group with trace minerals and vitamins $(G M T V)(n=$ $30)$ and group control $(G C)(n=30)$. There was no significant difference between those groups; however, we find significant difference between treatment and day for Superoxide dismutase (SOD) values with higher activity of this enzyme in females GMTV on the weeks next to the parturition. Still, there was difference on day and, for interactions between treatment and day for TBARS, were females GMTV showed lower values of TBARS in all moments, except on day seven after the parturition. For leucocytes, there is a significant effect by day, interaction on treatment and day on neutrophils, and interaction treatment and day for neutrophil phagocytosis, were females GMTV showed lower values of leucocytes and neutrophils next to the parturition, and an increase of neutrophils phagocytosis. In summary, cows from the GMTV group had an improvement on the immune and oxidative systems, probably correlated with the administration of this supplement.
\end{abstract}

Keywords: superoxide dismutase, gluationa peroxidase, TBARS, NBT, transition period

Recebido em 26 de dezembro de 2017

Aceito em 17 de maio de 2018

E-mail: silvana.collet@unoesc.edu.br 


\section{INTRODUÇÃO}

Nos últimos anos, as vacas leiteiras têm aumentado a produção de maneira significativa. Nesse sentido, observa-se o aumento de doenças metabólicas, principalmente, no período de transição. Durante essa fase, acontecem mudanças fisiológicas, metabólicas, nutricionais e hormonais. Estas são decorrentes de adaptações relacionadas com $\mathrm{o}$ aumento da demanda energética para suprir a glândula mamária, com os nutrientes necessários para a síntese do leite (Contreras e Sordillo, 2011). Além disso, esses animais ainda precisam lidar com a diminuição da ingestão de matéria seca e, por consequência, com o balanço energético negativo que, dependendo da intensidade, pode causar estresse oxidativo (Sordillo e Aitken, 2009).

O estresse oxidativo ocorre quando a produção de espécies reativas de oxigênio (ERO) e de espécies reativas de nitrogênio (ERN) é maior que a capacidade antioxidante do organismo. Este pode causar modificação em macromoléculas, morte celular, necrose e danos na estrutura dos tecidos (Spears e Weiss, 2008). Alguns estudos indicam o envolvimento do estresse oxidativo sobre o aparecimento de doenças em vacas leiteiras, estando envolvido em respostas disfuncionais do sistema imune e inflamatórias do animal, particularmente durante o período de transição (Sordillo, 2005; Sordillo e Aitken, 2009). Esse período também coincide com a redução das concentrações de vitaminas e de minerais que atuam como cofatores de algumas enzimas do sistema antioxidante, como a superóxido dismutase (SOD) e a glutationa peroxidase (GPx) (Xin et al.,1993).

Nesse sentido, o objetivo deste trabalho foi avaliar o efeito metafilático da administração subcutânea de minerais traços contendo cobre $(\mathrm{Cu})$, zinco $(\mathrm{Zn})$, selênio (Se), manganês $(\mathrm{Mn})$ e vitaminas A e E, em parâmetros do estresse oxidativo, no leucograma, no metabolismo oxidativo e fagocitose dos neutrófilos em vacas leiteiras no período de transição.

\section{MATERIAL E MÉTODOS}

O protocolo experimental foi aprovado pela Comissão de Ética em Uso de Animais da
Universidade Federal de Santa Maria (Ceua/UFSM), sob protocolo número 4688090815. Este estudo foi realizado em duas propriedades comerciais, nos municípios de Xanxerê-SC (propriedade 1) e Faxinal dos Guedes-SC (propriedade 2), com sistema de semiconfinamento. A propriedade 1 alojava 46 animais de peso médio de $630 \mathrm{~kg}$, com média de produção de 22 litros de leite por dia, e a propriedade 2 alojava 34 animais de peso médio $580 \mathrm{~kg}$, com média de produção de 24 litros de leite por dia. No período de 60 dias a 30 dias antes parto, os animais se alimentavam com volumoso e concentrado mineral comercial. No período pré-parto (30 dias antes do parto), os animais recebiam volumoso e concentrado aniônico comercial. No período de lactação, as vacas recebiam volumoso e concentrado. A composição química da dieta e a recomendação do National Research Council (Nutrient..., 2001) das dietas das propriedades estão descritas nas Tab. 1 e 2 .

Os animais foram divididos em dois grupos: grupo animais que receberam minerais traços e vitaminas A e E injetáveis (GMTV) $(n=30)$, e grupo controle (GC) $(n=30)$. A administração injetável mineral e vitamínica foi realizada por via subcutânea, com $5 \mathrm{~mL}$ do produto contendo $10 \mathrm{mg} / \mathrm{mL}$ de cobre (como edetato), $40 \mathrm{mg} / \mathrm{mL}$ de zinco (como edetato), $10 \mathrm{mg} / \mathrm{mL}$ de manganês (como edetato) e $5 \mathrm{mg} / \mathrm{mL}$ de selênio (como selenito de sódio) (Adaptador®min, cobre, zinco, selênio, manganês, Biogenesis Bagó, Argentina) e $5 \mathrm{~mL}$ do produto contendo $35 \mathrm{mg} / \mathrm{mL}$ de vitamina A (como palmitato) e $50 \mathrm{mg} / \mathrm{mL}$ de vitamina $\mathrm{E}$ (como acetato) (Adaptador $囚$ vit, vitamina $A$, vitamina $\mathrm{E}$, Biogenesis Bagó, Argentina). Os minerais e as vitaminas foram administrados aos 225 dias de gestação, 255 dias de gestação e no dia do parto. O grupo GC recebeu injeções de $5 \mathrm{~mL}$ de cloreto de sódio, $\mathrm{NaCl} 0,9 \%$, por via subcutânea, nos mesmos períodos. As coletas das amostras de sangue foram realizadas: M1 (21 dias pré-parto), M2 (14 dias pré-parto), M3 (sete dias pré-parto), M4 (dia do parto), M5 (sete dias após o parto), M6 (14 dias após o parto) e M7 (21 dias após o parto). 
Efeito do uso de minerais...

Tabela 1. Recomendação do National Research Council (Nutrient..., 2001) das dietas de vacas secas e em lactação e composição química da dieta total nos períodos pré-parto e lactação da propriedade 1

\begin{tabular}{|c|c|c|c|c|c|c|c|c|}
\hline & 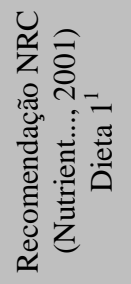 & 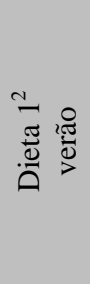 & $\begin{array}{l}n^{m} 0 \\
\stackrel{\Xi}{0} \\
\stackrel{\Xi}{0} \\
\stackrel{0}{0}\end{array}$ & 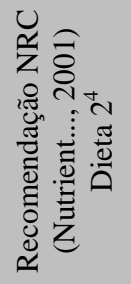 & 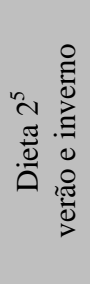 & 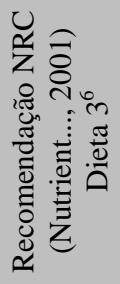 & 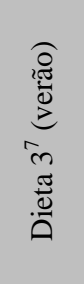 & 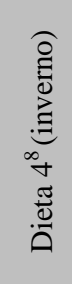 \\
\hline MS (\%) & 30 & 19,3 & 18,4 & 18,4 & 32,2 & 35 & 36,3 & 29,3 \\
\hline $\mathrm{PB}(\%)$ & 12 & 14,5 & 11 & 12 & 11,1 & 15 & 13,5 & 16,6 \\
\hline FDN (\%) & 33 & 29,1 & 66 & 33 & 42 & 33 & 37,4 & 37 \\
\hline FDA (\%) & 21 & 61,3 & 24,5 & 21 & 26,1 & 21 & 20,9 & 21 \\
\hline NDT (\%) & 51 & 55 & 55,3 & 51 & 69,6 & 66 & 68,9 & 73,5 \\
\hline CNF (\%) & Max.42 & 17,5 & 6,1 & Max.42 & 49 & 37 & 39,7 & 39,5 \\
\hline Amido (\%) & Max.25 & 7,6 & 1,0 & Max. 25 & 26 & 27 & 29,3 & 28 \\
\hline Lipídeo (\%) & . & 2,1 & 2,6 & & 2,6 & 3,5 & 2,4 & 2,6 \\
\hline $\mathrm{Cu}(\mathrm{mg} / \mathrm{kg})$ & 18 & 8 & 1 & 18 & 21 & 18 & 19 & 20 \\
\hline $\mathrm{Zn}(\mathrm{mg} / \mathrm{kg})$ & 43 & 65 & 1 & 43 & 26 & 43 & 28 & 35 \\
\hline $\mathrm{Se}(\mathrm{mg} / \mathrm{kg})$ & 0,3 & 0,5 & 0,01 & 0,3 & 0,66 & 0,3 & 0,56 & 57 \\
\hline $\mathrm{Mn}(\mathrm{mg} / \mathrm{kg})$ & 14 & 88 & 2 & 14 & 63 & 14 & 51 & 51 \\
\hline
\end{tabular}

${ }^{1}$ Recomendação NRC (Nutrient..., 2001) para dieta 60 aos 30 dias antes do parto. ${ }^{2}$ Dieta 60 aos 30 dias antes do parto verão. ${ }^{3}$ Dieta 60 aos 30 dias antes do parto inverno. ${ }^{4}$ Recomendação NRC (Nutrient..., 2001) para dieta 30 ao dia do parto. ${ }^{5}$ Dieta 30 ao dia do parto verão e inverno. ${ }^{6}$ Recomendação NRC (Nutrient..., 2001) para dieta lactação (vacas de peso médio de $630 \mathrm{~kg}$ e produzindo 22 litros de leite por dia). ${ }^{7}$ Dieta lactação verão. ${ }^{8}$ Dieta lactação inverno.

Tabela 2. Recomendação do National Research Council (NRC, 2001) das dietas de vacas secas e em lactação e composição química da dieta total nos períodos pré-parto e lactação da propriedade 2

\begin{tabular}{l}
\hline \\
\end{tabular}


A atividade sérica das enzimas glutationa peroxidase (GPx) e superóxido dismutase (SOD) foram determinadas em analisador bioquímico automático marca Randox ${ }^{\circledR}$ (modelo RX Daytona $\left.{ }^{\circledR}\right)$, utilizando-se kits comerciais Randox ${ }^{\circledR} \quad\left(\right.$ RANSOD $^{\circledR}$ e RANSEL $^{\circledR}$ ), como descrito por Woolliams et al. (1983) e Paglia e Valentine (1967), respectivamente. A peroxidação lipídica foi mensurada mediante produção de espécies reativas ao ácido tiobarbitúrico (TBARS), de acordo com técnica descrita por Ohkawa et al. (1979). O metabolismo oxidativo dos neutrófilos foi determinado pelo teste de redução do nitroazul de tetrazólio, estimulado ou não com partículas de Zymosan, por meio do uso de kit comercial, conforme método citoquímico descrito por Ciarlini et al. (2002). As avaliações do número total e diferencial de leucócitos foram realizadas por meio de técnicas de macrodiluição preconizadas por Weiss e Wardrop (2006).

O delineamento experimental foi em blocos ao acaso, sendo os tratamentos formados pelos grupamentos: grupo de animais que receberam minerais traços e vitaminas A e E injetáveis (GMTV) e grupo controle (GC). Os dados foram submetidos à análise de variância, com medidas repetidas no tempo dentro da variável aleatória vaca, utilizando-se o procedimento MIXED do pacote estatístico SAS, com estrutura de covariância autorregressiva, baseado no critério de informação de Akaike (AIC). Os dados foram previamente testados para normalidade dos resíduos pelo teste de Shapiro-Wilk. Foram consideradas significativas as diferenças ao nível de $5 \%$ de significância e tendência ao nível de $10 \%$.

\section{RESULTADOS E DISCUSSÃO}

Não houve diferença significativa do tratamento entre os grupos GMTV e GC para a atividade da enzima SOD ( $\mathrm{P}=0,1494)$. No entanto, observouse efeito significativo da interação entre tratamento e dia $(\mathrm{P}=0,0123)$ (Tab. 3). A enzima SOD exibiu maior atividade sete dias antes do parto e 14 dias após o parto no grupo de fêmeas GMTV (Fig. 1a), sendo, provavelmente, um reflexo da administração do $\mathrm{Cu}$ e do $\mathrm{Zn}$, que são cofatores funcionais da SOD. Sabe-se que o $\mathrm{Cu}$ e o Zn estão ligados em um mecanismo antioxidante envolvendo a enzima SOD, sendo esta responsável pela dismutação do radical superóxido, que é a primeira espécie reativa envolvida em processos oxidativos (Halliwell e Gutteridge, 2007). Machado et al. (2014) avaliaram o uso de injeções subcutâneas de um multimineral (contendo $\mathrm{Zn}$, Mn, Se e $\mathrm{Cu}$ ) e verificaram que houve aumento da atividade da enzima SOD até 100 dias após o parto nas fêmeas suplementadas, o que significa melhora na resposta antioxidante, assim como observado no presente estudo.

Quanto à enzima GPx não se observou diferença entre os tratamentos GMTV e GC $(\mathrm{P}=0,1650)$, porém constatou-se efeito significativo de dia $(\mathrm{P}=0,0366)$ (Tab. 3), quando houve redução da atividade dessa enzima no dia do parto, chegando ao menor nível na primeira semana após o parto, período em que ela se manteve em níveis mais baixos tanto em fêmeas GMTV quanto nas fêmeas GC (Fig. 1b). No entanto, o decréscimo na atividade da GPX foi mais acentuado nos animais do grupo controle (GC), fato que pode estar relacionado à administração injetável de Se. Nakov et al. (2016) também verificaram que houve redução na atividade sérica tanto da enzima GPx quanto da enzima SOD próximo ao parto, só ocorrendo aumento dela aos 21 dias de lactação. Aumentos nas concentrações de Se estão relacionados com maior atividade da enzima GPx, já que esse elemento é parte integrante dessa enzima (Rukgauer et al., 2001), a qual atua no sistema antioxidante celular convertendo peróxido de hidrogênio a água. Além de atuar no sistema antioxidante, o Se também está relacionado à melhora da função imune (Sordillo e Raphael, 2013). Weiss et al. (1990) realizaram pesquisa em rebanhos leiteiros comerciais e observaram que bovinos com altas concentrações de Se e de vitamina $\mathrm{E}$ no soro tiveram menor incidência de mastite clínica. De acordo com esses autores, esse efeito pode ser devido, em parte, à estreita ligação entre a vitamina $\mathrm{E}$ e o Se e a função dos neutrófilos.

Os dados referentes ao TBARS podem ser observados na Tab. 3. Não houve diferença significativa entre os tratamentos SMTV e GC $(\mathrm{P}=0,2059)$, mas houve efeito significativo para interação entre tratamento e dia $(\mathrm{P}=0,0482)$ e efeito significativo de dia $(\mathrm{P}=0,0018)$, os quais podem ser observados na Fig. 1c. Fêmeas SMTV mostraram valores médios menores de TBARS durante os momentos experimentais comparados às $\mathrm{GC}$, exceto aos sete dias, quando se observou um incremento nos valores do TBARS nas 
fêmeas GMTV. As substâncias reativas ao ácido tiobarbitúrico são um indicativo de peroxidação lipídica, e teores aumentados depois do parto podem indicar desbalanço entre subtâncias oxidantes e antioxidantes (Bouwstra et al., 2010). Chandra et al. (2013) relataram aumento de TBARS após o parto. Além disso, esses autores verificaram que a concentração de TBARS foi menor em vacas tratadas com vitamina $\mathrm{E}$ e $\mathrm{Zn}$ comparadas às vacas controle, fato que relacionaram à capacidade da vitamina $\mathrm{E}$ e do $\mathrm{Zn}$ em neutralizar a formação de ERO durante o pré e o pós-parto. No presente estudo, apesar de maiores valores numéricos do TBARS aos sete dias pós-parto no GMTV (Fig. 1c), observou-se que houve, nesse mesmo momento experimental, uma redução na atividade da SOD e GPx em ambos os grupos (Fig 1a. e 1b). No entanto, essa redução foi bem menos expressiva no GMTV, quando comparado ao GC.

Tabela 3. Médias dos quadrados mínimos \pm erros-padrão das médias (EPM) e valor de P para: superóxido dismutase (SOD) $(\mathrm{Ug} / \mathrm{Hb})$, glutationa peroxidase $(\mathrm{GPx})(\mathrm{Ug} / \mathrm{Hb})$ e teores de espécies reativas ao ácido tiobarbitúrico (TBARS (ņmol $\mathrm{MDA} / \mathrm{mL}$ ), em fêmeas Holandesas que receberam minerais traços e vitaminas A e E injetáveis (GMTV) e grupo controle (GC)

\begin{tabular}{|c|c|c|c|c|c|}
\hline Variável & Grupo & Médias e EPM & $P_{\text {Tratamento }}$ & $P_{\text {Dia }}$ & $P_{\text {Tratamento }} *$ Dia \\
\hline \multirow{2}{*}{ SOD } & GMTV & $3076,57 \pm 117,17 \mathrm{a}$ & \multirow{2}{*}{0,1494} & \multirow{2}{*}{0,7877} & \multirow{2}{*}{0,0123} \\
\hline & Controle & $2868,08 \pm 102,31 \mathrm{a}$ & & & \\
\hline \multirow{2}{*}{ GPx } & GMTV & $417,99 \pm 16,5048 \mathrm{a}$ & \multirow{2}{*}{0,1650} & \multirow{2}{*}{0,0366} & \multirow{2}{*}{0,9440} \\
\hline & Controle & $388,43 \pm 15,0065 \mathrm{a}$ & & & \\
\hline \multirow{2}{*}{ TBARS } & GMTV & $8,4872 \pm 0,8225 \mathrm{a}$ & \multirow{2}{*}{0,2059} & \multirow{2}{*}{0,0018} & \multirow{2}{*}{0,0482} \\
\hline & Controle & $9,8665 \pm 0,7120 \mathrm{a}$ & & & \\
\hline
\end{tabular}

Médias entre os grupos, seguidas por letras distintas, diferem entre si pelo teste de Tukey a 5\% de significância.

A avaliação de leucócitos totais e diferenciais pode ser visualizada na Tab. 4. Não houve diferença significativa entre os tratamentos GMTV e GC para leucócitos totais $(\mathrm{P}=0,1734)$, neutrófilos $(\mathrm{P}=0,1942)$, bastonetes $(0,2453)$, eosinófilos $(\mathrm{P}=0,1632)$, monócitos $(\mathrm{P}=0,8272)$, linfócitos $(\mathrm{P}=0,2614)$ e basófilos $(\mathrm{P}=0,6917)$. Apenas se observou efeito significativo de dia para leucócitos totais ( $\mathrm{P}=0,0093)$, em que, no dia do parto, constatou-se expressiva elevação nas fêmeas do GC, (Fig. 2d). Também houve efeito significativo de dia $(\mathrm{P}=0,0104)$ e para a interação tratamento e dia $(\mathrm{P}=0,0195)$ para neutrófilos (Fig. 1e), com elevação nas fêmeas GC, principalmente no dia do parto.

Segundo Sault e Birgel Júnior (2006), é sabido que, próximo ao parto, ocorrem intensas modificações no leucograma, sendo essas alterações caracterizadas por leucocitose devido à neutrofilia e à eosinopenia, decorrentes do aumento da secreção de hormônios da córtex adrenal, o que interfere na função imune desses animais, incluindo a função dos neutrófilos, a resposta dos linfócitos à estimulação mitogênica e a resposta de anticorpos (Kehrli et al., 2006). No presente estudo, pode-se observar que fêmeas do GC tiveram elevação de leucócitos e neutrófilos próximo ao parto, e isso demonstra que houve maior recrutamento de leucócitos e neutrófilos em relação às fêmeas SMTV (Fig. 1d e Fig. 1e). Esse fato pode estar diretamente ligado ao efeito dos minerais traços e vitaminas A e E, sendo esses elementos capazes de modular a resposta imune nas fêmeas GMTV. Torre et al. (1996) verificaram que neutrófilos de novilhas alimentadas com dieta deficiente em $\mathrm{Cu}$ apresentaram redução da morte de Staplylococcus aureus aproximadamente 35 dias pós-parto. Cebra et al. (2003) observaram que neutrófilos obtidos de vacas com alta concentração sanguínea de Se tinham maior potencial para produzir superóxido e matar bactérias patogênicas. Hogan et al. (1992) verificaram que a administração oral ou parenteral de vitamina $\mathrm{E}$ em vacas leiteiras periparturientes não afetou a atividade fagocítica dos neutrófilos, mas melhorou sua capacidade em matar bactérias. 
Os resultados referentes ao metabolismo oxidativo e à fagocitose dos neutrófilos podem ser visualizados na Tab. 5. Não houve diferença entre os tratamentos GMTV e GC para metabolismo oxidativo basal $(\mathrm{P}=0,2495)$, metabolismo oxidativo estimulado $(\mathrm{P}=0,1098)$, fagocitose de neutrófilos $(\mathrm{P}=0,7010)$ nem para fagocitose de neutrófilos com metabolismo ativo $(0,4199)$. Porém, observou-se efeito da interação tratamento e dia para fagocitose de neutrófilos com metabolismo ativo $(\mathrm{P}=0,0462)$, em que fêmeas GMTV tiveram maior fagocitose por neutrófilos no dia do parto e sete dias após o parto (Fig. 2f), sugerindo que o uso dos minerais traços e das vitaminas A e E melhorou a função imune dessas fêmeas nesse período.

Boyne e Arthur (1979) avaliaram a função de neutrófilos pelo teste de NBT em bezerros da raça Holandesa e verificaram que o grupo de animais com dieta deficiente em selênio tinha atividade inferior ao grupo com dieta com teores de selênio adequada. Fonquete et al. (2013) avaliaram o leucograma e o metabolismo oxidativo de neutrófilos em cabras da raça Saanen e não detectaram diferença no metabolismo oxidativo dos neutrófilos nos períodos de gestação, parto e pós-parto. A redução do NBT tem uma estreita correlação com o metabolismo oxidativo e avalia um importante componente da atividade bactericida do neutrófilo (Roth et al., 1983). A maior produção de substâncias oxidativas no interior do neutrófilo, ocasionada pelo aumento da atividade respiratória dessa célula, é importante para a adequada eficiência desse processo de defesa (Tizard, 2000). Dessa forma, pode-se observar maior atividade de neutrófilos medidos pelo NBT em fêmeas do grupo GMTV, e isto, associado ao menor número de leucócitos e neutrófilos nesse mesmo período, confere melhor imunidade a esses animais.

Tabela 4. Médias dos quadrados mínimos \pm erros-padrão das médias (EPM) e valor de P para: leucócitos totais, neutrófilos, bastonetes, eosinófilos, linfócitos, monócitos e basófilos, em fêmeas Holandesas que receberam minerais traços e vitaminas A e E injetáveis (GMTV) e grupo controle (GC)

\begin{tabular}{|c|c|c|c|c|c|}
\hline Variável & Grupo & Médias e EPM & $\mathrm{P}_{\text {Tratamento }}$ & $P_{\text {Dia }}$ & $P_{\text {Tratamento } * \text { Dia }}$ \\
\hline \multirow[b]{2}{*}{ Leucócitos totais } & GMTV & $8576,65 \pm 532,98 \mathrm{a}$ & \multirow[b]{2}{*}{0,1734} & \multirow[b]{2}{*}{0,0093} & \multirow[b]{2}{*}{0,3604} \\
\hline & Controle & $9644,54 \pm 604,44 \mathrm{a}$ & & & \\
\hline \multirow{2}{*}{ Neutrófilos } & GMTV & $1929,86 \mathrm{a}$ & \multirow{2}{*}{0,1942} & \multirow{2}{*}{0,0104} & \multirow{2}{*}{0,0195} \\
\hline & Controle & $2212,12 \mathrm{a}$ & & & \\
\hline Bastonetes & $\begin{array}{l}\text { GMTV } \\
\text { Controle }\end{array}$ & $\begin{array}{c}513 \pm 68,2763 \mathrm{a} \\
617,16 \pm 60,0595 \mathrm{a}\end{array}$ & 0,2453 & 0,157 & 0,7476 \\
\hline Eosinófilos & $\begin{array}{l}\text { GMTV } \\
\text { Controle }\end{array}$ & $\begin{array}{l}360,59 \pm 45,2555 \mathrm{a} \\
443,64 \pm 39,3602 \mathrm{a}\end{array}$ & 0,1632 & 0,3024 & 0,8213 \\
\hline Monócitos & $\begin{array}{l}\text { GMTV } \\
\text { Controle }\end{array}$ & $\begin{array}{l}1033,00 \pm 75,83 \mathrm{a} \\
1011,75 \pm 61,70 \mathrm{a}\end{array}$ & 0,8272 & 0,3622 & 0,6852 \\
\hline \multirow{2}{*}{ Linfócitos } & GMTV & $4604,00 \pm 436,65 \mathrm{a}$ & \multirow[b]{2}{*}{0,2614} & \multirow[b]{2}{*}{0,0843} & \multirow[b]{2}{*}{0,6110} \\
\hline & Controle & $5241,47 \pm 395,10 \mathrm{a}$ & & & \\
\hline Basófilos & $\begin{array}{l}\text { GMTV } \\
\text { Controle }\end{array}$ & $\begin{array}{l}180,32 \pm 22,6703 \mathrm{a} \\
192,21 \pm 19,7348 \mathrm{a}\end{array}$ & 0,6917 & 0,5028 & 0,1920 \\
\hline
\end{tabular}

Médias entre os grupos, seguidas por letras distintas, diferem entre si pelo teste de Tukey a 5\% de significância. 


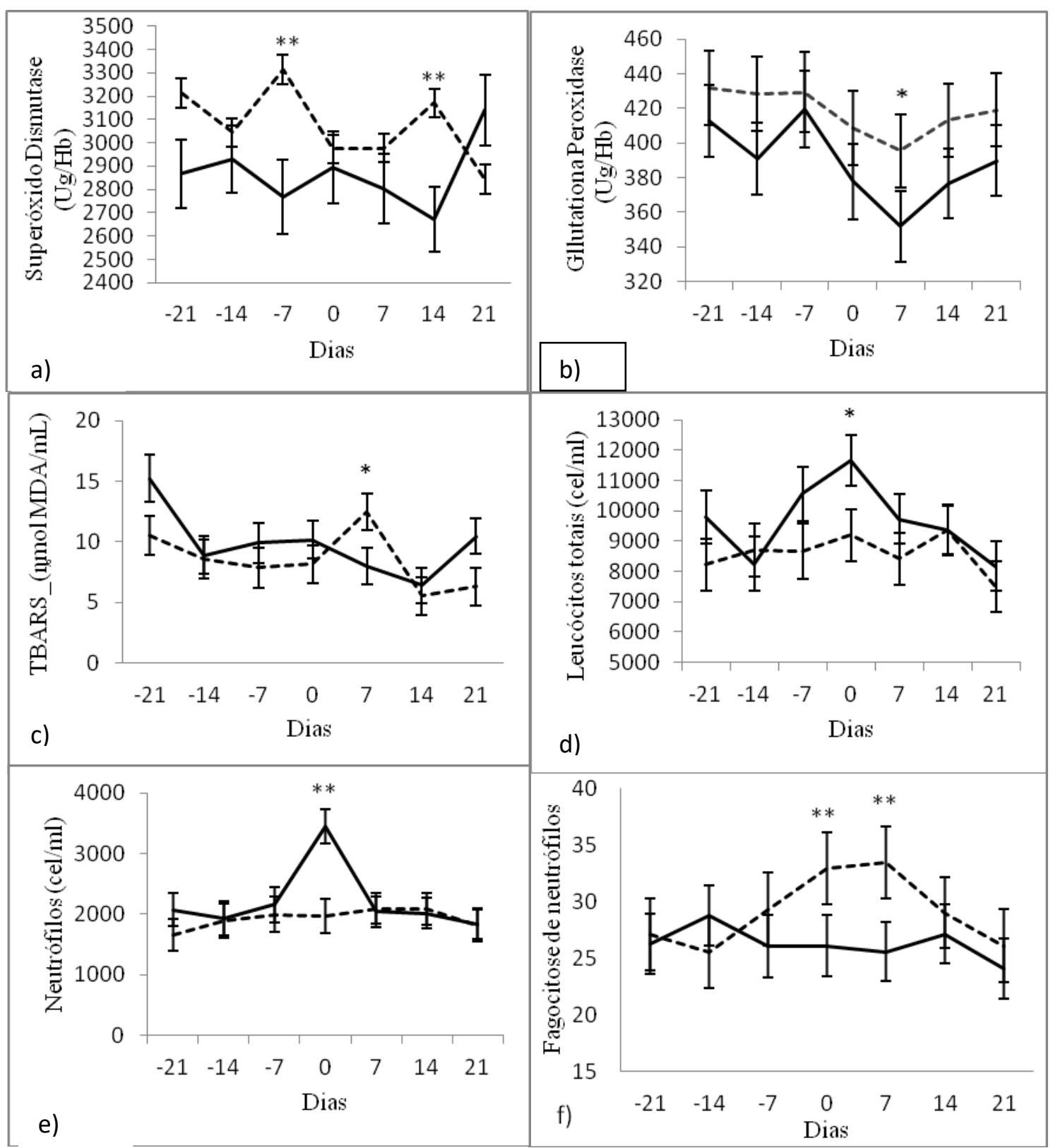

Figura 1. a) atividade da enzima superóxido dismutase (SOD (Ug/Hb) em função das semanas em lactação em fêmeas dos grupos GMTV (- - ) ou GC (-); b) atividade da enzima glutationa peroxidase (GPx (Ug/Hb) em função das semanas em lactação em fêmeas dos grupos GMTV (- - -) ou GC( - ); c) produção de espécies reativas ao ácido tiobarbitúrico (TBARS) (nomol MDA/mL) em função das semanas em lactação em fêmeas GMTV (- - -) ou GC(-); d) leucócitos totais (cel/mL) em função das semanas em lactação em fêmeas GMTV (- - -) ou GC (-); e) neutrófilos (cel/mL) em função das semanas em lactação em fêmeas GMTV (- - -) ou GC (-); f) fagocitose de neutrófilos com metabolismo ativo em função das semanas em lactação em fêmeas GMTV (- - ou GC (-). A barra de erros representa o padrão de erro da média. * Efeito significativo de dia. ** Efeito significativo entre interação tratamento e dia. 
Tabela 5. Médias dos quadrados mínimos \pm erros-padrão das médias (EPM) e valor de $\mathrm{P}$ para: metabolismo oxidativo basal, metabolismo oxidativo estimulado, fagocitose de neutrófilos e fagocitose e metabolismo oxidativo, em fêmeas Holandesas que receberam minerais traços e vitaminas A e E injetáveis (GMTV) e grupo controle (GC)

\begin{tabular}{|c|c|c|c|c|c|}
\hline Variável & Grupo & Médias e EPM & $P_{\text {Tratamento }}$ & $P_{D i a}$ & $P_{\text {Tratamento*Dia }}$ \\
\hline $\begin{array}{l}\text { Metabolismo oxidativo } \\
\text { basal }\end{array}$ & $\begin{array}{l}\text { GMTV } \\
\text { C }\end{array}$ & $\begin{array}{l}53,0820 \pm 2,5135 \mathrm{a} \\
56,7478 \pm 1,9833 \mathrm{a}\end{array}$ & 0,2495 & 0,1219 & 0,4691 \\
\hline $\begin{array}{l}\text { Metabolismo oxidativo } \\
\text { estimulado }\end{array}$ & $\begin{array}{c}\text { GMTV } \\
\text { C }\end{array}$ & $\begin{array}{l}21,0165 \pm 1,6250 \mathrm{a} \\
21,0165 \pm 1,6250 \mathrm{a}\end{array}$ & 0,1098 & 0,1924 & 0,5305 \\
\hline \multirow[t]{2}{*}{$\begin{array}{l}\text { Fagocitose de } \\
\text { neutrófilos }\end{array}$} & $\begin{array}{c}\text { GMTV } \\
\text { C }\end{array}$ & $\begin{array}{l}26,5300 \pm 2,1221 a \\
25,5049 \pm 1,6623 a\end{array}$ & 0,7010 & 0,1389 & 0,5513 \\
\hline & GMTV & $29,0363 \pm 2,7298 a$ & & & \\
\hline $\begin{array}{l}\text { Fagocitose e } \\
\text { metabolismo oxidativo }\end{array}$ & $\mathrm{C}$ & $26,2700 \pm 2,1317 \mathrm{a}$ & 0,4199 & 0,2048 & 0,0462 \\
\hline
\end{tabular}

Médias entre os grupos, seguidas por letras distintas, diferem entre si pelo teste de Tukey a 5\% de significância.

\section{CONCLUSÃO}

Em conclusão, animais com dietas adequadas e moderada produção leiteira apresentaram pouca resposta ao uso de metafiláticos. No entanto, observou-se com esse estudo que fêmeas que receberam administração subcutânea com minerais traços e vitaminas $\mathrm{A}$ e $\mathrm{E}$ tiveram incremento da enzima SOD e fagocitose de neutrófilos com metabolismo ativo. Isso reforça a ligação entre minerais traços e vitaminas ao sistema imune de bovinos, especialmente no período de transição, o qual está ligado à diminuída resposta imune.

\section{REFERÊNCIAS}

BOUWSTRA, R.J.; NIELEN, M.; NEWBOLD, J.R.; JANSEN, E.H. et al. Vitamin E supplementation during the dry period in dairy cattle. Part II: Oxidative stress following vitamin E supplementation may increase clinical mastitis incidence postpartum. J. Dairy Sci., v.93, p.5696-5706, 2010.

BOYNE, R.; ARTHUR, J.R. Alterations of neutrophil function in selenium-deficient cattle. J. Comp. Patoh., v.89, p.151, 1979.

CEBRA, C.K.; HEIDEL, J.R.; CRISMAN, R.O.; STANG, B.V. Relationship between endogenous cortisol, blood micronutrients, and netrophil function in postparturient Holstein cows. J. Vet. Int. Med., v.17, p.902-907, 2003.
CHANDRA, G.; AGGARWAL, A.; SINGH, A.K. KUMAR, M. Effect of vitamin E and zinc supplementation on energy metabolites, lipid peroxidation, and milk production in peripartum sahiwal cows. Asian-Aust. J. Anim. Sci., v.26, p.1569-1576, 2013.

CIARLINI, P.C.; CIARLINI, L.D.R.P.; ALENCAR, N.X. et al. Metabolismo oxidativo de neutrófilos em ovelhas naturalmente infectadas por nematóides gastrointestinais e correlação com o nível sérico de cortisol e carga parasitária. Arq. Bras. Med. Vet. Zoot., v.54, p.17, 2002.

CONTRERAS, G.A.; SORDILLO, L.M. Lipid mobilization and inflammatory responses during the transition period of dairy cows. Comp. Immunol. Microbiol. Infec. Dis., v.34, p.281-289, 2011.

FONQUETE, J.H.; SAITO, M.R.; BARIONI, G.; VALENTE, A.C.S.; TAKAHIRA, R.K.; KOHAYAGAWA, A. Leucograma e metabolismo oxidativo de neutrófilos em cabras da raça Saanen nos períodos de gestação, parto e pós-parto. Pesqui. Vet. Bras., v.33, p.63-70, 2013.

HALLIWELL, B.; GUTTERIDGE, J.M.C. Free radicals in biology and medicine. Oxford: Clarendon Press, 2007. 851p.

HOGAN, J.S.; WEISS, W.P.; TODHUNTER, D.A. et al. Bovine neutrophil responses to parenteral vitamin E. J. Dairy Sci., v.73, p.399405,1992 . 
KEHRLI JR., M.E.; NEILL, J.D.; BURVENICH, C. et al. Energy and protein effects on the immune system. In: SEJRSEN, K.; HVELPLUND, T.; NIELSEN, M.O. (Eds.). Ruminant Physiology, 2006. p.455-471.

MACHADO, V.S.; OIKONOMOU, G.; LIMA, S.F. et al. The effect of injectable trace minerals (selenium, copper, zinc, and manganese) on peripheral blood leukocyte activity and serum superoxide dismutase activity of lactating Holstein cows. Vet. J., v.200, p.299-304, 2014.

NAKOV, D.; ANDONOV, S.; TRAJCHEV, M. Antioxidant status in dairy cows during transition period. J. Agric. Food Env. Sci., v.68, p.1-8, 2016.

NATIONAL RESEARCH COUNCIL - NRC. Nutrient requeriments of dairy cattle. 7.rev.ed. Washinton, D.C.: 2001. 381p.

OHKAWA, H.; OHISHI, N.; YAGI, K. Assay for lipid peroxides in animal tissues by thiobarbituric acid reaction. Anal. Biochem., v.95, p.351-358,1979.

PAGLIA, D.E.; VALENTINE W.N. Studies on the quantitative and qualitative characterization of erythrocyte glutathione peroxidase. J. Lab. Clin Med., v.70, p.158-169, 1967.

RIKGAUER, M.; NEUGEBAUER, R. J.; PLECKO, T. The relation between selenium, zinc and copper concentration and the trace element dependent antioxidative status. J. Trace Elem. Med. Biol., v.15, p.73-78, 2001.

ROTH, J.A.; KAEBERLE, M.L.; APPELL L.H.; NACHREINER, R.F. Association of increased estradiol and progesterone blood values with altered bovine polymorphonuclear leukocyte function. Am. J. Vet. Res., v.44, p.247-253, 1983.

SAULT, J.P.E.; BIRGEL JUNIOR, E.H. Influência do período pós-parto sobre o leucograma de fêmeas bovinas da raça holandesa. Braz. J. Vet. Res. Anim. Sci., v.43, p.588-597, 2006.
SPEARS, J. W.; W. P. WEISS. Role of antioxidants and trace elements in health and immunity of transition dairy cows. The Vet. J., v. 176, p. 70-76, 2008.

SORDILLO, L.M. Factors affecting mammary gland immunity and mastitis susceptibility. Livest. Prod. Sci., v.98, p.89-99, 2005.

SORDILLO, L.M.; AITKEN, S.L. Impact of oxidative stress on the health and immune function of dairy cattle. Vet. Immunol. Immunopathol., v.128, p.104-109, 2009.

SORDILLO, L.M.; RAPHAEL, W. Significance of metabolic stress, lipid mobilization, and inflammation on transition cow disorders. Vet. Clin. N. Am. Food Prac., v.29, p.267-278, 2013.

TIZARD, I.R. (Ed.). Veterinary immunology: an introduction. London: Saunders Company, 2000. 482p.

TORRE, P.M.; HARMON, R.J.; HEMKEN, R.W. et al. Mild dietary copper insufficiency depresses blood neutrophil function in dairy cattle. J. Nut. Immol., v.4, p.3-24, 1996.

WEISS, D.J.; WARDROP, J.K. Schalm's veterinary hematology. Philadelphia: Lea \& Febiger, 2006. 1344p.

WEISS, W.P.; HOGAN, J.S.; SMITH, K.L. et al. Relationships among selenium, vitamin E, and mammary gland health in commercial dairy herds. J. Dairy Sci., v.73, p.81, 1990.

WOOLLIAMS, J.A.; WIENER, G.; ANDERSON, P.H. et al. Variation in the activities of glutathione peroxidase and superoxide dismutase and in the concentration of copper in the blood in various breed crosses of sheep. Res. Vet. Sci., v.34, p.253-256, 1983

XIN, Z.; WATERMAN, D.F.; HEMKEN, R.W. et al. Copper status and requirement during the dry period and early lactation in multiparous Holstein cows. J. Dairy Sci., v.76, p.2711-2716, 1993. 